TRANSACTIONS OF THE

AMERICAN MATHEMATICAL SOCIETY

Volume 354, Number 7, Pages 2971-2982

S 0002-9947(02)02968-9

Article electronically published on March 5, 2002

\title{
A NON-FIXED POINT THEOREM FOR HAMILTONIAN LIE GROUP ACTIONS
}

\author{
CHRISTOPHER ALLDAY, VOLKER HAUSCHILD, AND VOLKER PUPPE
}

\begin{abstract}
We prove that, under certain conditions, if a compact connected Lie group acts effectively on a closed manifold, then there is no fixed point. Because two of the main conditions are satisfied by any Hamiltonian action on a closed symplectic manifold, the theorem applies nicely to such actions. The method of proof, however, is cohomological; and so the result applies more generally.
\end{abstract}

\section{INTRODUCTION}

In the cohomology theory of transformation groups (based on a study of the Borel construction) most theorems concern torus actions, and results about nonabelian Lie group actions are scarce. In this paper we shall give two results about compact connected Lie group actions: the first gives a condition under which a compact connected Lie group $G$ cannot act effectively on a certain type of space; and the second, which is a small variation on the first, gives a condition under which $G$ cannot act effectively with a fixed point.

Both results apply to Hamiltonian actions on closed symplectic manifolds; but, since the proof is purely cohomological, they apply rather more generally. The main condition imposed on the space $X$, on which $G$ acts, concerns the degrees of the relations in an algebra presentation of the rational or real cohomology algebra of $X$. This kind of information is often well known for symplectic manifolds, especially for toric varieties: see, e.g., Audin, Chapter VI, Theorem 4.4.3. It is also known for some symplectic quotients: see [Tolman,Weitsman].

One of our main results is the following (Corollary (4.8) below).

Theorem. Let $G$ be a compact connected Lie group and let $M$ be a closed symplectic manifold. For a field $k$ of characteristic 0 , suppose $H^{*}(M ; k)$ has a $k$-algebra presentation

$$
0 \longrightarrow J \longrightarrow k\left[x_{1}, \ldots, x_{g}\right] \otimes \wedge\left(y_{1}, \ldots, y_{h}\right) \longrightarrow H^{*}(M ; k) \longrightarrow 0,
$$

where $x_{i}, 1 \leq i \leq g$, and $y_{i}, 1 \leq i \leq h$, are generators of even degree, respectively, odd degree; and the ideal $J=\left(f_{1}, \ldots, f_{m}, e_{1}, \ldots, e_{n}\right)$, where $f_{i}, 1 \leq i \leq m$, and $e_{i}$, $1 \leq i \leq n$, are relations of even degree, respectively, odd degree. Let $H^{*}(B G ; k)=$ $k\left[v_{1}, \ldots, v_{r}\right]$.

Received by the editors November 4, 2001.

2000 Mathematics Subject Classification. Primary 57S15; Secondary 53D99, 55N91, 57R17.

Key words and phrases. Compact connected Lie group actions, Hamiltonian actions, fixed points, cohomology theory. 
Suppose that

$$
\max \left\{\operatorname{deg}\left(f_{i}\right) ; 1 \leq i \leq m\right\} \leq \max \left\{\operatorname{deg}\left(v_{i}\right) ; 1 \leq i \leq r\right\} .
$$

Suppose that $G$ is acting on $M$ in an effective Hamiltonian way. Then there is no fixed point.

\section{Preliminaries}

Throughout this paper $k$ will denote a field of characteristic 0. Recall the following notation and terminology used in Allday,Puppe.

Definitions (2.1). (1) If $G$ is a compact Lie group, then $G^{0}$ denotes the connected component of the identity.

(2) Let $G$ be a compact Lie group and let $X$ be a paracompact $G$-space. Then $X$ is said to satisfy Condition (LT) if either

(a) $X$ is compact; or

(b) the set of conjugacy classes of identity components of isotropy subgroups, $\left\{\left[G_{x}^{0}\right] ; x \in X\right\}$, is finite, and

(i) the cohomological dimension of $X$ over $k, c d_{k}(X)$, is finite, or

(ii) $X$ is finitistic.

(For some comments on the finitistic (Swan) condition see Allday,Puppe. For some information on cohomological dimension see Bredon2] or [Quillen. Note that spaces of finite covering dimension, such as finite-dimensional $C W$-complexes and finite-dimensional manifolds, are finitistic. Note too that, by a theorem of [Mann, the set of conjugacy classes of isotropy subgroups, $\left\{\left[G_{x}\right] ; x \in X\right\}$, is finite whenever $G$ is a compact Lie group and $X$ is an orientable topological manifold such that $H_{*}(X ; \mathbb{Z})$ is finitely generated.)

(3) Let $G$ be a compact Lie group and let $X$ be a $G$-space. Let $X \rightarrow^{i_{G}} X_{G}$ $\rightarrow^{p} B G$ be the fibre bundle of the Borel construction. Then $X$ is said to be totally non-homologous to zero (TNHZ) in $X_{G}$ with respect to $H^{*}(-; k)$ if the Leray-Serre spectral sequence of $p$ in $H^{*}(-; k)$ collapses, i.e. $E_{2}=E_{\infty}$, or, equivalently,

$$
i_{G}^{*}: H_{G}^{*}(X ; k) \longrightarrow H^{*}(X ; k)
$$

is surjective.

Next we need to recall two standard variants on the notion of effectiveness.

Definitions (2.2). Let $G$ be a compact connected Lie group and let $X$ be a $G^{-}$ space.

(1) The action of $G$ on $X$ is said to be almost-effective if the ineffective kernel, $\bigcap_{x \in X} G_{x}$, is finite.

(2) The action of $G$ on $X$ is said to be cohomologically effective (with coefficients in $k$ ) if the restriction homomorphism

$$
H^{*}(X ; k) \longrightarrow H^{*}\left(X^{K} ; k\right)
$$

is not a monomorphism for any subcircle $K \subseteq G$. Otherwise the action is said to be cohomologically ineffective. (In Allday,Puppe, Definition (4.7.3), the condition is that the restriction homomorphism is not an isomorphism for any subcircle $K \subseteq G$. This is equivalent to the monomorphism condition used here if $X$ satisfies Condition (LT).) 
(3) The action of $G$ on $X$ is said to be cohomologically trivial if the restriction homorphism

$$
H^{*}(X ; k) \longrightarrow H^{*}\left(X^{G} ; k\right)
$$

is an isomorphism.

Remarks (2.3). (1) Any non-trivial action of a simple compact connected Lie group is almost-effective.

(2) If $G$ is a compact connected Lie group and $X$ is a closed orientable manifold, then an action of $G$ on $X$ is cohomologically effective if and only if it is almost-effective. More generally, this holds if $X$ is a compact orientable cohomology manifold over $k$. (See Borel et al., Chapter I, Corollary 4.6, and Chapter V, Theorem 3.2.)

For more details on the Borel construction see, for example, Allday,Puppe, Atiyah,Bott, Borel et al., Bredon3], Guillemin,Sternberg, [Hsiang or Quillen.

\section{Hamiltonian and Cohomologically Hamiltonian Actions}

As before, $k$ will denote a field of characteristic 0 .

Definitions (3.1). Let $X$ be a space, and let $G$ be a compact connected Lie group. Suppose that $X$ satisfies Poincaré duality with coefficients in $k$, and suppose that the formal dimension of $X, f d(X)$, is $2 n$ : i.e., $H^{i}(X ; k)=0$ for $i>2 n, H^{2 n}(X ; k) \cong$ $k, \operatorname{dim}_{k} H^{i}(X ; k)<\infty$, for all $i$, and the cup product pairing

$$
H^{i}(X ; k) \times H^{2 n-i}(X ; k) \longrightarrow H^{2 n}(X ; k) \cong k
$$

is non-singular for all $i$.

(1) $X$ is said to be cohomologically symplectic (c-symplectic) if there is a class $w \in H^{2}(X ; k)$ such that $w^{n} \neq 0 . w$ is called the $c$-symplectic class.

(2) If $G$ is acting on $X$, then the action is said to be cohomologically Hamiltonian $(c$-Hamiltonian) if

$$
w \in i m\left[i_{G}^{*}: H_{G}^{*}(X ; k) \longrightarrow H^{*}(X ; k)\right],
$$

where, as before, $i_{G}: X \longrightarrow X_{G}$ is the inclusion of the fibre in the Borel construction bundle $X_{G} \longrightarrow B G$.

Remarks (3.2). (1) A closed symplectic manifold is $c$-symplectic (over $\mathbb{R}$ ) with $w=[\omega]$, the class of the symplectic form.

(2) For an action of a connected compact Lie group $G$ on a $c$-symplectic space $X$, it always holds that $g^{*} w=w$, for all $g \in G$; and so any action of $G$ is said to be $c$-symplectic.

(3) If $M$ is a closed symplectic manifold, $G$ is a compact connected Lie group, $G$ is acting on $M$, and the action is symplectic, then the action is Hamiltonian if and only if it is $c$-Hamiltonian. This follows directly from the Cartan model for computing equivariant cohomology: see Guillemin,Sternberg. See also Audin, Chapter V, Proposition 3.1.1.

(4) See Allday2 or Lupton,Oprea for more about actions on $c$-symplectic spaces.

(5) If $X$ satisfies Poincaré duality over $k$ with $f d(X)=2 n$, and if there are classes $w_{1}, \ldots, w_{n} \in H^{2}(X ; k)$ such that $w_{1} \ldots w_{n} \neq 0$, then $X$ is $c$-symplectic. (This follows because char $k=0$.) In particular, suppose that $X$ is a topological 
manifold, or, more generally, a rational cohomology manifold, and suppose that $\operatorname{dim}_{\mathbb{Q}} H^{i}(X ; \mathbb{Q})<\infty$ for all $i$. Then $X$ is $c$-symplectic over a field $k$ of characteristic 0 if and only if $X$ is $c$-symplectic over $\mathbb{Q}$.

The next lemma is important for our main results.

Lemma (3.3). Let $X$ be a c-symplectic space satisfying Condition (LT)- see Definitions (2.1)(2). Let $G=S^{1}$, the circle group, and suppose that $G$ is acting on $X$ in a $c$-Hamiltonian and cohomologically effective way. Then the fixed point set $X^{G}$ is non-empty and it has at least two connected components.

See Allday2, Proposition (6.7) and Remark (6.8) for the easy proof; and see Theorem (6.10), there, for the generalization to torus actions. The result is, of course, easy for truly Hamiltonian actions since the moment map must have a maximum and a minimum.

We also want the following theorem of Frankel. Since Frankel's proof was ostensibly only for circle actions, we shall indicate how to deduce the theorem as stated here.

Theorem (3.4) ([Frankel]). Let $M$ be a closed symplectic manifold, let $G$ be a compact connected Lie group, and suppose that $G$ is acting on $M$ in a Hamiltonian way. Then $M$ is TNHZ in $M_{G} \longrightarrow B G$ with respect to $H^{*}(-; k)$.

When $G=S^{1}$, Frankel uses Morse theory to prove that

$$
\operatorname{dim}_{k} H^{*}(M ; k)=\operatorname{dim}_{k} H^{*}\left(M^{G} ; k\right) .
$$

By a standard result in the cohomology theory of transformation groups, this is equivalent to $M$ being TNHZ in $M_{G} \longrightarrow B G$ with respect to $H^{*}(-; k)$. See, e.g., Allday,Puppe, Theorem (3.10.4). The following proposition, then, shows how to generalize Frankel's theorem to all compact connected Lie groups. The proof is an easy matter of comparing spectral sequences.

Proposition (3.5). Let $G$ be a compact connected Lie group, and let $X$ be a $G-$ space. Then the following statements are equivalent.

(1) $X$ is TNHZ in $X \rightarrow^{i_{G}} X_{G} \longrightarrow B G$ with respect to $H^{*}(-; k)$.

(2) For any compact connected Lie group $K$ and homomorphism $\varphi: K \longrightarrow G, X$ is TNHZ in $X \rightarrow^{i_{K}} X_{K} \longrightarrow B K$ with respect to $H^{*}(-; k)$, where $X$ is made into a $K$-space via $\varphi$.

(3) For every subcircle $L \subseteq G, X$ is $T N H Z$ in $X \rightarrow^{{ }^{L}} X_{L} \longrightarrow B L$ with respect to $H^{*}(-; k)$.

(4) $X$ is TNHZ in $X \rightarrow^{i_{T}} X_{T} \longrightarrow B T$ with respect to $H^{*}(-; k)$ for at least one maximal torus $T \subseteq G$.

Remarks (3.6). (1) Frankel's theorem does not hold in general for $c$-Hamiltonian actions, even with the weak Lefschetz condition. See Allday1 for an example. By an old result of Blanchard, however, the theorem holds for any compact connected Lie group action on a $c$-Kähler space, i.e., a $c$-symplectic space satisfying the strong (or hard) Lefschetz condition. See [Blanchard] or Borel et al.], Chapter XII, Section 6.

(2) Since TNHZ implies $c$-Hamiltonian, the converse of Frankel's theorem is valid by Remarks (3.2)(3).

(3) By Remarks (3.2)(3) and Proposition (3.5), if $M$ is a closed symplectic manifold and $G$ is a compact connected Lie group acting on $M$ in a symplectic way, then the following statements are equivalent. 
(i) The action of $G$ is Hamiltonian.

(ii) The action of at least one maximal subtorus $T \subseteq G$ is Hamiltonian.

(iii) The action of every maximal subtorus $T \subseteq G$ is Hamiltonian.

(iv) The action of every subcircle $L \subseteq G$ is Hamiltonian.

(4) Since the condition TNHZ is equivalent to the vanishing of the differential in the appropriate minimal Hirsch-Brown model (see Allday,Puppe, Definitions (3.5.6) et seq.), one can restate part of Proposition (3.5) as follows.

The differential in the minimal Hirsch-Brown model $H^{*}(B G ; k) \otimes H^{*}(X ; k)$ is zero if and only if the differential in the minimal Hirsch-Brown model $H^{*}(B L ; k) \otimes$ $H^{*}(X ; k)$ is zero for every subcircle $L \subseteq G$.

\section{The Main Theorem}

In the following let $R_{G}=H^{*}(B G ; k)$, where, as always here, $k$ is a field of characteristic zero. Furthermore, $k$ is considered as an $R_{G}$-module via the standard augmentation homomorphism $R_{G} \longrightarrow k$.

We begin with an easy generalization of a result of T. Chang ( Chang $)$. Chang's proof works without change here.

Lemma (4.1). Let $X$ be a space, and suppose that $H^{*}(X ; k)$ has a $k$-algebra presentation

$$
0 \longrightarrow J \longrightarrow k\left[x_{1}, \ldots, x_{g}\right] \otimes \wedge\left(y_{1}, \ldots, y_{h}\right) \longrightarrow H^{*}(X ; k) \longrightarrow 0,
$$

where $x_{1}, \ldots, x_{g}$ are generators of positive even degree, $y_{1}, \ldots, y_{h}$ are generators of odd degree, and the ideal of relations $J=\left(f_{1}, \ldots, f_{m}, e_{1}, \ldots, e_{n}\right)$, where $f_{1}, \ldots, f_{m}$ are relations of even degree and $e_{1}, \ldots, e_{n}$ are relations of odd degree.

Let $G$ be a compact connected Lie group, and suppose that $G$ is acting on $X$ so that $X$ is TNHZ in $X_{G} \longrightarrow B G$ with respect to $H^{*}(-; k)$.

Then $H_{G}^{*}(X ; k)$ has an $R_{G}$-algebra presentation as follows, and there is a commutative diagram

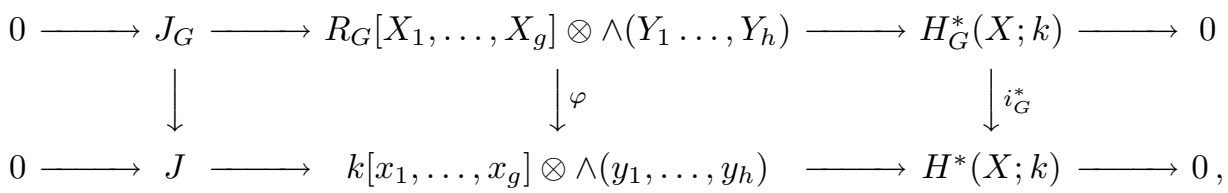

where $J_{G}=\left(F_{1}, \ldots, F_{m}, E_{1}, \ldots, E_{n}\right), \varphi\left(X_{i}\right)=x_{i}$ for $1 \leq i \leq g, \varphi\left(Y_{i}\right)=y_{i}$ for $1 \leq i \leq h, \varphi\left(F_{i}\right)=f_{i}$ for $1 \leq i \leq m$ and $\varphi\left(E_{i}\right)=e_{i}$ for $1 \leq i \leq n$.

Next we need a version of the Evaluation Theorem, Allday,Puppe, Theorem (3.5.1). Let $X$ be a connected space, $k$ a field of characteristic 0 , and $T$ a torus, and suppose that $T$ is acting on $X$ so that $X$ is TNHZ in $X_{T} \longrightarrow B T$. Let $R_{T}=H^{*}(B T ; k)=k\left[t_{1}, \ldots, t_{r}\right]$, where $r=\operatorname{rank}(T)$ and $\operatorname{deg}\left(t_{i}\right)=2$, for $1 \leq i \leq r$.

Let $\mathfrak{p} \subseteq R_{T}$ be a homogeneous prime ideal. Then there is a field $E$ extending $k$, and $\alpha_{1}, \ldots, \alpha_{r} \in E$, such that the evaluation homomorphism $\alpha: R_{T} \longrightarrow E[t]$, defined by $\alpha\left(t_{i}\right)=\alpha_{i} t$ for $1 \leq i \leq r$, has kernel $\mathfrak{p}$ : i.e., $\operatorname{ker}(\alpha)=\mathfrak{p}$. As in Allday,Puppe, Section 3.5, we define the subtorus $K(\alpha) \subseteq T$ to be the smallest subtorus of $T$ with $P K(\alpha) \subseteq \mathfrak{p}$, where, for a subtorus $K \subseteq T, P K$ is the kernel of the restriction homomorphism $R_{T} \longrightarrow R_{K}$. So $K(\alpha)$ is non-trivial provided that $\mathfrak{p} \neq\left(t_{1}, \ldots, t_{r}\right)$, the maximal homogeneous ideal in $R_{T}$.

We have the following version of Allday,Puppe, Theorem (3.5.1). 
Lemma (4.2). Let $X, k, T$ and the action be as above. (In particular, $X$ is TNHZ in $X_{T} \longrightarrow B T$.) Suppose that $X$ satisfies Condition (LT): see Definitions (2.1)(2). Let $\mathfrak{p}, \alpha$ and $K(\alpha)$ be as above. Then $\alpha$ factors through $R_{K(\alpha)}=H^{*}(B K(\alpha) ; k)$ to give $\bar{\alpha}: R_{K(\alpha)} \longrightarrow E[t]$. (So $\alpha$ is the composition of $\bar{\alpha}$ with the epimorphism $\left.R_{T} \longrightarrow R_{K(\alpha)} \cdot\right)$ Also, there are isomorphisms

$$
\begin{aligned}
E[t] \otimes_{\alpha} H_{T}^{*}(X ; k) & \cong E[t] \otimes_{\bar{\alpha}} H_{K(\alpha)}^{*}(X ; k) \\
E\left[t, t^{-1}\right] \otimes_{\alpha} H_{T}^{*}(X ; k) & \cong E\left[t, t^{-1}\right] \otimes H^{*}\left(X^{K(\alpha)} ; k\right) .
\end{aligned}
$$

Proof. Since $X$ is TNHZ in $X_{T} \longrightarrow B T, H_{T}^{*}(X ; k)$ is a free $R_{T}$-module; and so, from the pull-back diagram

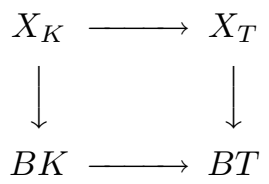

for any subtorus $K \subseteq T$, using either the Leray-Serre spectral sequence or the Eilenberg-Moore spectral sequence,

$$
H_{K}^{*}(X ; k) \cong R_{K} \otimes_{R_{T}} H_{T}^{*}(X ; k) .
$$

The first, unlocalized, part above follows immediately.

The rest follows as in the proof of Allday,Puppe, Theorem (3.5.1), noting that $S(K(\alpha)) \cap \operatorname{ker}(\alpha)=\emptyset$, where $S(K(\alpha))$ is the multiplicative subset of $R_{T}$ generated by all homogeneous linear polynomials not in $P K(\alpha)$, and, hence, not in $\operatorname{ker}(\alpha)$.

Remark (4.3). The proof of Lemma (4.2) shows that

$$
E[t] \otimes_{\alpha} H_{T}^{*}(X ; k) \cong E[t] \otimes_{\bar{\alpha}} H_{K}^{*}(X ; k),
$$

for any subtorus $K \subseteq T$ such that $K(\alpha) \subseteq K$. Furthermore, Condition (LT) is not needed for this isomorphism. But, if $K(\alpha) \underset{\neq}{\subset} K$, then $S(K) \cap \operatorname{ker}(\alpha) \neq \emptyset$; and the localized part of Lemma (4.2) is not valid.

Corollary (4.4). With the notation and conditions of Lemma (4.2), suppose, in addition, that $E[t] \otimes_{\alpha} H_{T}^{*}(X ; k)$ is generated, as an $E[t]-$ algebra, by nilpotent elements. Then $X^{K}$ is connected for every subtorus $K \subseteq K(\alpha)$.

Proof. Since the action is uniform (see Allday,Puppe, Corollary (3.6.19)), it is enough to prove the result when $K=K(\alpha)$. Suppose that $X^{K}$ is not connected: let $F_{1} \ldots, F_{s}$ be its components. Let $e=(1,0, \ldots, 0) \in \bigoplus_{i=1}^{s} H^{0}\left(F_{i} ; k\right)=H^{0}\left(X^{K} ; k\right)$, and let $e^{\prime}=1-e=(0,1, \ldots, 1)$. By Lemma (4.2), there is a non-negative integer $m$, and there are elements $u, u^{\prime} \in E[t] \otimes_{\alpha} H_{T}^{*}(X ; k)$, such that $\varphi^{*}(u)=t^{m} e$ and $\varphi^{*}\left(u^{\prime}\right)=t^{m} e^{\prime}$, where $\varphi^{*}$ is the standard homomorphism

$$
E[t] \otimes_{\alpha} H_{T}^{*}(X ; k) \stackrel{\sim}{\longrightarrow} E[t] \otimes_{\bar{\alpha}} H_{K}^{*}(X ; k) \longrightarrow E[t] \otimes_{\bar{\alpha}} H_{K}^{*}\left(X^{K} ; k\right) .
$$

(Note that $E[t] \otimes_{\bar{\alpha}} H_{K}^{*}\left(X^{K} ; k\right) \cong E[t] \otimes H^{*}\left(X^{K} ; k\right)$.)

Since $\varphi^{*}\left(u u^{\prime}\right)=0$, it follows from Lemma (4.2) that $u u^{\prime}=0$. But $u$ and $u^{\prime}$ are non-nilpotent, since $e$ and $e^{\prime}$ are non-nilpotent. So $u u^{\prime}=0$ contradicts the assumption that $E[t] \otimes_{\alpha} H_{T}^{*}(X ; k)$ is generated by nilpotent elements as an $E[t]-$ algebra.

In the following, for a Noetherian $\operatorname{ring} A, \operatorname{dim}(A)$ means the Krull dimension. 
Corollary (4.5). Assume the notation and conditions of Lemma (4.1), suppose that $G=T$, a torus, and suppose that $X$ satisfies Condition (LT). Consider $f_{i}=$ $1 \otimes f_{i}$, for $1 \leq i \leq m$, as an element in $R_{T}\left[X_{1}, \ldots, X_{g}\right] \otimes \wedge\left(Y_{1}, \ldots, Y_{h}\right)$ via the standard lifting. Let $\mathfrak{a} \subseteq R_{T}$ be the ideal generated by the coefficients (in $R_{T}$ ) of the polynomials $F_{i}-f_{i}$ for $1 \leq i \leq m$. Suppose that $\operatorname{dim}\left(R_{T} / \mathfrak{a}\right)>0$. Then there is a subcircle $K \subseteq T$ such that $X^{K}$ is connected.

Proof. Since $\operatorname{dim}\left(R_{T} / \mathfrak{a}\right)>0$, there is a minimal prime ideal $\mathfrak{p}$ of $\mathfrak{a}$ such that $\mathfrak{p} \underset{\neq}{\subsetneq}$ $\left(t_{1}, \ldots, t_{r}\right)$, the maximal homogeneous ideal of $R_{T}$. Let $\alpha$ be as in Lemma (4.2). So $K(\alpha)$ is non-trivial.

Since $X$ satisfies Condition (LT), $H^{*}(X ; k)$ is generated by nilpotent elements. But, since $\mathfrak{a} \subseteq \mathfrak{p}=\operatorname{ker}(\alpha)$,

$$
E[t] \otimes_{\alpha} H_{T}^{*}(X ; k) \cong E[t]\left[X_{1}, \ldots, X_{g}\right] \otimes \wedge\left(Y_{1}, \ldots, Y_{h}\right) / J_{\alpha},
$$

where $J_{\alpha}=E[t] \otimes_{\alpha} J_{T}$. So the even generators of $J_{\alpha}$ are $f_{1}, \ldots, f_{m}$. Thus it follows that $E[t] \otimes_{\alpha} H_{T}^{*}(X ; k)$ is generated by nilpotent elements as an $E[t]-$ algebra. Hence $X^{K}$ is connected for any subtorus $K \subseteq K(\alpha)$.

The following is our main technical result.

Theorem (4.6). Assume the notation and conditions of Lemma (4.1), and suppose that $X$ satisfies Condition (LT) for a maximal torus $T \subseteq G$.

Let $R_{G}=H^{*}(B G ; k)=k\left[v_{1}, \ldots, v_{r}\right]$, where $r=\operatorname{rank}(G)$. Suppose either

(1) $\max \left\{\operatorname{deg}\left(f_{i}\right) ; 1 \leq i \leq m\right\}<\max \left\{\operatorname{deg}\left(v_{i}\right) ; 1 \leq i \leq r\right\}$; or

(2) $X^{G} \neq \emptyset$ and

$$
\max \left\{\operatorname{deg}\left(f_{i}\right) ; 1 \leq i \leq m\right\} \leq \max \left\{\operatorname{deg}\left(v_{i}\right) ; 1 \leq i \leq r\right\} .
$$

Then there is a subcircle $K \subseteq T$ such that $X^{K}$ is connected.

Proof. By a standard result in equivariant cohomology, see, e.g., Allday,Puppe, Theorem $(3.9 .3)(2)$,

$$
H_{T}^{*}(X ; k) \cong R_{T} \otimes_{R_{G}} H_{G}^{*}(X ; k) .
$$

Thus $H_{T}^{*}(X ; k)$ has an algebra presentation with exactly the same generators and relations as $H_{G}^{*}(X ; k)$.

Let $\mathfrak{a}$ be the ideal defined in Corollary (4.5).

Now assume condition (1) on the degrees, and suppose that $v_{1}, \ldots, v_{r}$ are ordered so that $\operatorname{deg}\left(v_{1}\right) \leq \cdots \leq \operatorname{deg}\left(v_{r}\right)$. So $\mathfrak{a} \subseteq\left(v_{1}, \ldots, v_{r-1}\right)$.

It follows from Krull's Theorem (see, e.g., Allday,Puppe, Theorem (A.1.5)) that $\operatorname{dim}\left(R_{T} / \mathfrak{a}\right)>0$. So the result in this case follows from Corollary (4.5).

Now assume condition (2). Let $\sigma: B G \longrightarrow X_{G}$ be a section of the bundle $X_{G} \longrightarrow B G$ coming from a fixed point. Let $\bar{X}_{i}$ be the image of $X_{i}$ in $H_{G}^{*}(X ; k)$ for $1 \leq i \leq g$. Subtracting elements of $R_{G}$ if necessary, we can choose $X_{i}$ so that $\sigma^{*}\left(\bar{X}_{i}\right)=0$ for $1 \leq i \leq g$.

Let $\epsilon: R_{G}\left[X_{1}, \ldots, X_{g}\right] \otimes \wedge\left(Y_{1}, \ldots, Y_{h}\right) \longrightarrow R_{G}$ be the standard augmentation given by $\epsilon\left(X_{i}\right)=0$ for $1 \leq i \leq g$. Since $\sigma^{*}\left(\bar{X}_{i}\right)=0$ for $1 \leq i \leq g, \epsilon=\sigma^{*} q$, where $q: R_{G}\left[X_{1}, \ldots, X_{g}\right] \otimes \wedge\left(Y_{1}, \ldots, Y_{h}\right) \longrightarrow H_{G}^{*}(X ; k)$ is the quotient homomorphism. Clearly $\epsilon\left(f_{i}\right)=0$ for $1 \leq i \leq m$; and, of course, $q\left(F_{i}\right)=0$ for $1 \leq i \leq m$. (Here $f_{i}$ and $F_{i}$ are as in Corollary (4.5).) So $\epsilon\left(F_{i}-f_{i}\right)=0$ for $1 \leq i \leq m$. Hence the coefficients of $F_{i}-f_{i}$ have degrees less than $\operatorname{deg}\left(F_{i}-f_{i}\right)$. Thus condition (2) implies that $\mathfrak{a} \subseteq\left(v_{1}, \ldots, v_{r-1}\right)$. The result now follows as before. 
We shall now give three corollaries and some examples.

Corollary (4.7). Let $G$ be a compact connected Lie group and let $M$ be a csymplectic $G$-space. ( $M$ is c-symplectic with respect to a given field $k$ of characteristic 0.) Suppose that $M$ satisfies Condition (LT) for a maximal torus $T \subseteq G$.

Suppose that $H^{*}(M ; k)$ has a $k$-algebra presentation

$$
0 \longrightarrow J \longrightarrow k\left[x_{1}, \ldots, x_{g}\right] \otimes \wedge\left(y_{1}, \ldots, y_{h}\right) \longrightarrow H^{*}(M ; k) \longrightarrow 0
$$

where $x_{i}$ has positive even degree for $1 \leq i \leq g$, yi has odd degree for $1 \leq i \leq h$, and the ideal of relations $J=\left(f_{1}, \ldots, f_{m}, e_{1}, \ldots, e_{n}\right)$, where $f_{i}$ is a relation of even degree for $1 \leq i \leq m$, and $e_{i}$ is a relation of odd degree for $1 \leq i \leq n$.

Let $R_{G}=H^{*}(B G ; k)=k\left[v_{1}, \ldots, v_{r}\right]$, where $r=\operatorname{rank}(G)$.

Suppose that $M$ is TNHZ in $M_{T} \longrightarrow B T$ (or, equivalently, in $M_{G} \longrightarrow B G$ ), with respect to $H^{*}(-; k)$, and suppose that the action of $T$ on $M$ is c-effective. Finally, suppose that

$$
\max \left\{\operatorname{deg}\left(f_{i}\right) ; 1 \leq i \leq m\right\} \leq \max \left\{\operatorname{deg}\left(v_{i}\right) ; 1 \leq i \leq r\right\} .
$$

Then $M^{G}=\emptyset$.

Proof. If $M^{G} \neq \emptyset$, then, by Theorem (4.6), there is a subcircle $K \subseteq T$ such that $M^{K}$ is connected. This contradicts Lemma (3.3).

Corollary (4.8). Let $G$ be a compact connected Lie group and let $M$ be a closed symplectic manifold. For a field $k$ of characteristic 0 , suppose $H^{*}(M ; k)$ has a $k$-algebra presentation

$$
0 \longrightarrow J \longrightarrow k\left[x_{1}, \ldots, x_{g}\right] \otimes \wedge\left(y_{1}, \ldots, y_{h}\right) \longrightarrow H^{*}(M ; k) \longrightarrow 0
$$

where $J=\left(f_{1}, \ldots, f_{m}, e_{1}, \ldots, e_{n}\right)$, and $x_{i}, 1 \leq i \leq g, y_{i}, 1 \leq i \leq h, f_{i}, 1 \leq i \leq m$, and $e_{i}, 1 \leq i \leq n$, are as in Corollary (4.7).

Let $R_{G}=H^{*}(B G ; k)=k\left[v_{1}, \ldots, v_{r}\right]$, and suppose that

$$
\max \left\{\operatorname{deg}\left(f_{i}\right) ; 1 \leq i \leq m\right\} \leq \max \left\{\operatorname{deg}\left(v_{i}\right) ; 1 \leq i \leq r\right\} .
$$

Suppose that $G$ is acting on $M$ in an almost-effective Hamiltonian way. Then $M^{G}=\emptyset$.

Proof. The action is $c$-effective by Remarks (2.3)(2), and $M$ is TNHZ in $M_{G} \longrightarrow$ $B G$ by Frankel's Theorem (3.4). So the result follows from Corollary (4.7).

The next corollary is an explicit application to certain homogeneous spaces which are Kählerian.

Corollary (4.9). Let $G$ be a compact connected Lie group, and let $M=G / Z(K)$, where $K \subseteq G$ is a (not necessarily maximal) subtorus, and $Z(K)$ is the centralizer of $K$ in $G$. Suppose that $G$ is acting on $M$ in any continuous almost-effective way. Then $M^{G}=\emptyset$.

Proof. Let $f_{1}, \ldots, f_{m}, v_{1}, \ldots, v_{r}$ be as in Corollary (4.7). From the fibre bundle $G \longrightarrow M \longrightarrow B Z(K)$, it follows that $m=r$ and

$$
H^{*}(M ; \mathbb{Q}) \cong \mathbb{Q}\left[u_{1}, \ldots, u_{r}\right] /\left(f_{1}, \ldots, f_{r}\right)
$$

where $\mathbb{Q}\left[u_{1}, \ldots, u_{r}\right]=H^{*}(B Z(K) ; \mathbb{Q})$ and $\operatorname{deg}\left(f_{i}\right)=\operatorname{deg}\left(v_{i}\right)$ for $1 \leq i \leq r$.

By a result of Borel (see [Tralle,Oprea, Chapter 5, Section 2), $M$ is a Kähler manifold. Hence $M$ is TNHZ in $M_{G} \longrightarrow B G$ (which also follows easily since $H^{*}(M ; \mathbb{Q})$ is zero in odd degrees). So the result follows from Corollary (4.7). 
Examples (4.10). (1) Let

$$
M=U(n) / U\left(n_{1}\right) \times \cdots \times U\left(n_{s}\right) \approx S U(n) / S\left(U\left(n_{1}\right) \times \cdots \times U\left(n_{s}\right)\right),
$$

where $n_{1}+\cdots+n_{s}=n$. Then no non-trivial action of $S U(n)$ on $M$ has a fixed point. This follows from Corollary (4.9). (See Remarks (2.3)(1).)

(2) Let $M=S^{2} \times S^{2} \times \cdots \times S^{2}=\left(S^{2}\right)^{n}$, or, more generally, let $M$ be any space satisfying Condition (LT) and having rational cohomology isomorphic to that of $\left(S^{2}\right)^{n}$. Let $G$ be any non-abelian compact connected Lie group. If $G$ acts almosteffectively (or, in the general case, cohomologically effectively) on $M$, then $M^{G}=\emptyset$.

Since $G$ is non-abelian, $H^{*}(B G ; \mathbb{Q})$ has a generator of degree 4 . So this example follows from Corollary (4.7).

\section{Generalizations}

In Corollaries (4.7) and (4.8), the crucial point is that effective Hamiltonian circle actions do not have connected fixed point sets. (See Lemma (3.3).) In this section we briefly consider a generalization through the notion of minimal formal dimension and the related notion of minimal grading (cf. [Hauschild]). The definitions are as follows.

Definitions (5.1). Let $k$ be a field, and let $A^{*}$ be a connected commutative graded $k$-algebra. Suppose that the total dimension of $A^{*}$, as a $k$-vector space, is finite. Let $A^{(*)}$ denote the same algebra viewed as $\mathbb{Z}_{2}$-graded: i.e., all elements of even degree are considered to have degree 0 , and all elements of odd degree are considered to have degree 1. Let

$\mathcal{A}=\left\{B^{*} ; B^{*}\right.$ is a connected commutative graded $k$ - algebra and $\left.B^{(*)} \cong A^{(*)}\right\}$.

(1) $A^{*}$ is said to have minimal grading if, given any $B^{*} \in \mathcal{A}$,

$$
\sum_{i=0}^{\infty} \operatorname{dim}_{k} B^{m+2 i} \leq \sum_{i=0}^{\infty} \operatorname{dim}_{k} A^{m+2 i} \text { for all } m \geq 0
$$

implies that

$$
\operatorname{dim}_{k} B^{j}=\operatorname{dim}_{k} A^{j} \text { for all } j .
$$

(2) Suppose that $A^{*}$ satisfies Poincaré duality over $k$. Then $A^{*}$ is said to have minimal formal dimension if $f d\left(A^{*}\right) \leq f d\left(B^{*}\right)$ for all $B^{*} \in \mathcal{A}$.

Remarks (5.2). (1) A Poincaré duality algebra has minimal grading if it has minimal formal dimension.

(2) If $X$ is a $c$-symplectic space (over a field $k$ of characteristic 0 ), then $H^{*}(X ; k)$ has minimal formal dimension (and hence also minimal grading).

(3) For $B^{*} \in \mathcal{A}$, define the dimension vector of $B^{*}$ to be

$$
d\left(B^{*}\right)=\left(\operatorname{dim}_{k} B^{\circ}, \operatorname{dim}_{k} B^{1}, \ldots\right),
$$

i.e., the sequence of coefficients in the Poincaré series of $B^{*}$. Suppose that $d\left(A^{*}\right)$ is maximal among all $d\left(B^{*}\right)$ for $B^{*} \in \mathcal{A}$ with respect to the lexicographic order: i.e., for any $B^{*} \in \mathcal{A}$, either

(i) $\operatorname{dim}_{k} B^{j}=\operatorname{dim}_{k} A^{j}$ for all $j \geq 0$, or

(ii) there is a positive integer $j$ such that $\operatorname{dim}_{k} B^{i}=\operatorname{dim}_{k} A^{i}$ for all $i<j$, and $\operatorname{dim}_{k} B^{j}<\operatorname{dim}_{k} A^{j}$.

Then $A^{*}$ has minimal grading. 
An algebra with minimal grading, however, need not have a maximal dimension vector: consider, for example, the rational cohomology of the connected sum $\mathbb{C} P^{4} \#\left(S^{4} \times S^{4}\right)$ compared to that of $\mathbb{C} P^{4} \#\left(S^{2} \times S^{6}\right)$.

In Remarks (2.3)(2), it was pointed out that the notions of almost-effective and cohomologically effective (over a field $k$ of characteristic 0 ) are equivalent for compact orientable cohomology manifolds over $k$ (or, more simply, for closed orientable topological manifolds). The next theorem relates cohomological effectiveness directly to formal dimension: it is due to Bredon ([Bredon1]) and Chang and Skjelbred ([Chang,Skjelbred1]). The assertion in the last paragraph is not given explicitly in these two references; but it is an easy consequence of the first paragraph and the Localization Theorem.

Theorem (5.3). Let $T$ be a torus, $X$ a T-space and $k$ a field of characteristic 0 . Suppose that $X$ satisfies Condition (LT), and that $X^{T} \neq \emptyset$. Suppose that $X$ satisfies Poincaré duality over $k$. Let $F$ be a component of $X^{T}$. Then $F$ satisfies Poincaré duality over $k$, and $f d(F) \leq f d(X)$.

Furthermore, if $f d(F)=f d(X)$, then $F=X^{T}$, and the restriction homomorphism $H^{*}(X ; k) \longrightarrow H^{*}(F ; k)$ is an isomorphism: i.e., the action is cohomologically trivial (see Definitions (2.2)(3)).

We can now state the following partial generalization of Corollary (4.7). It is necessary here to include the relations of odd degree as well as those of even degree. In many applications, however, there are no generators of odd degree (and hence no relations of odd degree).

Theorem (5.4). Assume the notation and conditions of Lemma (4.1), and suppose that $X$ satisfies Condition (LT) for a maximal torus $T \subseteq G$. Suppose further that either (1) $H^{*}(X ; k)$ satisfies Poincaré duality with minimal formal dimension, or (2) $H^{*}(X ; k)$ has minimal grading.

Let $R_{G}=H^{*}(B G ; k)=k\left[v_{1}, \ldots, v_{r}\right]$, where $r=\operatorname{rank}(G)$; and suppose that

$$
\begin{aligned}
& \max \left\{\operatorname{deg}\left(f_{i}\right) ; 1 \leq i \leq m\right\} \leq \max \left\{\operatorname{deg}\left(v_{i}\right) ; 1 \leq i \leq r\right\}, \\
& \max \left\{\operatorname{deg}\left(e_{i}\right) ; 1 \leq i \leq n\right\} \leq \max \left\{\operatorname{deg}\left(v_{i}\right) ; 1 \leq i \leq r\right\} .
\end{aligned}
$$

Suppose that the action of $G$ on $X$ is cohomologically effective.

Then $X^{G}=\emptyset$.

Proof. Suppose that $X^{G} \neq \emptyset$. As in Corollary (4.5),

$$
E[t] \otimes_{\alpha} H_{T}^{*}(X ; k) \cong E[t]\left[X_{1}, \ldots, X_{g}\right] \otimes \wedge\left(Y_{1}, \ldots, Y_{h}\right) / J_{\alpha},
$$

where now $J_{\alpha}=\left(f_{1} \ldots, f_{m}, e_{1}, \ldots, e_{n}\right)$, where in $e_{i}$ the variables $x_{j}, y_{j}$ have been replaced by $X_{j}, Y_{j}$. So

$$
E[t] \otimes_{\alpha} H_{T}^{*}(X ; k) \cong E[t] \otimes H^{*}(X ; k)
$$

as $E[t]$-algebras.

Putting $t=1$ in Lemma (4.2), we now see that

$$
H^{(*)}(X ; k) \cong H^{(*)}\left(X^{K(\alpha)} ; k\right)
$$

as $\mathbb{Z}_{2}$-graded $k$-algebras.

In case (1), minimal formal dimension, the result now follows (by contradiction) from Theorem (5.3). 
Now consider case (2), minimal grading. Let $K=K(\alpha)$, and let $\varphi: X^{K} \longrightarrow$ $X$ be the inclusion. By the dimensional inequalities (see, e.g., Allday,Puppe, Corollary (3.1.14) or Theorem (3.10.13)),

$$
\sum_{i=0}^{\infty} \operatorname{dim}_{k} H^{m+2 i}\left(X^{K} ; k\right) \leq \sum_{i=0}^{\infty} \operatorname{dim}_{k} H^{m+2 i}(X ; k),
$$

for any $m \geq 0$.

Thus the minimal grading condition implies that

$$
\operatorname{dim}_{k} H^{j}\left(X^{K} ; k\right)=\operatorname{dim}_{k} H^{j}(X ; k),
$$

for all $j \geq 0$. And it now follows from the Localization Theorem that $\varphi^{*}: H^{*}(X ; k)$ $\longrightarrow H^{*}\left(X^{K} ; k\right)$ is an isomorphism, which contradicts the cohomological effectiveness.

\section{REFERENCES}

[Allday1] C. Allday, Examples of circle actions on symplectic spaces, Homotopy and Geometry, J. Oprea and A. Tralle, eds., Banach Center Publications 45, 87-90, Polish Academy of Sciences, Warsaw, 1998. MR 99k:57001

[Allday2] C. Allday, Notes on the Localization Theorem with applications to symplectic torus actions, Proceedings of the Winter School on Transformation Groups, Indian Statistical Institute, Calcutta 1998, to appear.

[Allday,Puppe] C. Allday and V. Puppe, Cohomological Methods in Transformation Groups, Cambridge Studies in Advanced Mathematics 32, Cambridge University Press, Cambridge, 1993. MR 94g:55009

[Atiyah,Bott] M. Atiyah and R. Bott, The moment map and equivariant cohomology, Topology 23 (1984), 1-28. MR 85e:58041

[Audin] M. Audin, The Topology of Torus Actions on Symplectic Manifolds, Progress in Mathematics 93, Birkhäuser-Verlag, Basel, Boston, Berlin, 1991. MR 92m:57046

[Blanchard] A. Blanchard, Sur les variétés analytiques complexes, Annales Ecole Norm. Sup. 73 (1957), 157-202. MR 19:316e

[Borel et al.] A. Borel et al., Seminar on Transformation Groups, Ann. of Math. Studies 46, Princeton University Press, Princeton, 1960. MR 22:7129

[Bredon1] G. Bredon, Fixed point sets of actions on Poincaré duality spaces, Topology 12 (1973), 159-175. MR 48:9708

[Bredon2] G. Bredon, Sheaf Theory, Second Edition, Graduate Texts in Mathematics 170, Springer-Verlag, New York, 1997. MR 98g:55005

[Bredon3] G. Bredon, Introduction to Compact Transformation Groups, Academic Press, New York (1972). MR 54:1265

[Chang] T. Chang, On the number of relations in the cohomologyof a fixed point set, Manuscript Math. 18 (1976), 237-247. MR 54:13954

[Chang,Skjelbred1] T. Chang and T. Skjelbred, Group actions on Poincaré duality spaces, Bull. Amer. Math. Soc. 78 (1972), 1024-1026. MR 46:6346

[Chang,Skjelbred2] T. Chang and T. Skjelbred, Lie group actions on a Cayley projective plane and a note on homogeneous spaces of prime Euler Characteristic, Amer. J. Math. 98 (1976), 655-678. MR 55:1371

[Frankel] T. Frankel, Fixed points and torsion on Kähler manifolds, Ann. of Math. 70 (1959), 1-8. MR 24:A1730

[Guillemin,Sternberg] V. Guillemin and S. Sternberg, Supersymmetry and Equivariant de Rham Theory, Springer-Verlag, Berlin, Heidelberg, 1999. MR 2001i:53140

[Hauschild] V. Hauschild, Transformation groups on complex Grassmannians, to appear.

[Hsiang] W.-Y. Hsiang, Cohomology Theory of Topological Transformation Groups, Ergebnisse der Math. und ihrer Grenzgebiete 85, Springer-Verlag, New York, Heidelberg, Berlin, 1975. MR 54:11363 
[Lupton,Oprea] G. Lupton and J. Oprea, Cohomologically symplectic spaces: toral actions and the Gottlieb group, Trans. Amer. Math. Soc. 347 (1995), 261-288. MR 95f:57056

[Mann] L. Mann, Finite orbit structure on locally compact manifolds, Mich. Math. J. 9 (1962), 87-92. MR 24:A1966

[Quillen] D. Quillen, The spectrum of an equivariant cohomology ring: I, Ann. of Math. 94 (1971), 549-572. MR 45:7743

[Tolman,Weitsman] S. Tolman and J. Weitsman, The cohomologyrings of Abelian symplectic quotients, xxx.lanl.gov/math.DG/9807173, July 1998. MR 98k:53038

[Tralle,Oprea] A. Tralle and J. Oprea, Symplectic Manifolds with no Kähler Structure, Lecture Notes in Math. 1661, Springer-Verlag, Berlin, Heidelberg, New York, 1997.

Department of Mathematics, University of Hawail at Manoa, Honolulu, Hawail 96822 2273

E-mail address: chris@math.hawaii.edu

Department of Mathematics, University of Calabria, I-87036 Rende, Italy

E-mail address: hausch@unical.it

Faculty of Mathematics, University of Konstanz, D-78457 Konstanz, Germany

E-mail address: volker.puppe@uni-konstanz.de 